\title{
Solvation Structure and Dynamics of Alkali Metal Halides in Ionic Liquid from Classical Molecular Dynamics Simulations
}

Rahul Gupta, Thejus R. Kartha and Bhabani S. Mallik*

Department of Chemistry, Indian Institute of Technology Hyderabad, Kandi-502285,

Sangareddy, Telangana, India

Table S1. Non-bonded Force Field Parameters for [Bmim][OTf]

\begin{tabular}{|c|c|c|}
\hline Atom type & $\boldsymbol{\sigma}(\AA)$ & $\boldsymbol{\varepsilon} \mathbf{( k J ~ m o l}^{\mathbf{- 1}} \mathbf{)}$ \\
\hline C3 & 0.339967 & 0.45773 \\
\hline CC & 0.339967 & 0.359824 \\
\hline CD & 0.339967 & 0.359824 \\
\hline H & 0.3250 & 0.71128 \\
\hline H1 & 0.247135 & 0.0656888 \\
\hline H4 & 0.251055 & 0.06276 \\
\hline HC & 0.242146 & 0.06276 \\
\hline F & 0.264953 & 0.0656888 \\
\hline O & 0.311815 & 0.255224 \\
\hline S6 & 0.295992 & 0.87864 \\
\hline & 0.356359 & 1.0460 \\
\hline
\end{tabular}

Table S2. Force field parameters for single ions of alkali metal halides

\begin{tabular}{|c|c|c|}
\hline Ion & $\boldsymbol{\sigma}(\AA)$ & $\boldsymbol{\varepsilon}\left(\mathbf{k J} \mathbf{~ m o l}^{-1}\right)$ \\
\hline $\mathrm{Li}^{+}$ & 2.02590 & $7.65672 \times 10^{-2}$ \\
\hline $\mathrm{Na}^{+}$ & 3.32840 & $1.15897 \times 10^{-2}$ \\
\hline $\mathrm{K}^{+}$ & 4.73602 & $1.37235 \times 10^{-3}$ \\
\hline $\mathrm{Rb}^{+}$ & 5.26699 & $7.11280 \times 10^{-4}$ \\
\hline $\mathrm{Cs}^{+}$ & 6.04920 & $3.37230 \times 10^{-4}$ \\
\hline $\mathrm{F}^{-}$ & 3.11815 & $2.55224 \times 10^{-1}$ \\
\hline $\mathrm{Cl}^{-}$ & 4.40104 & $4.18400 \times 10^{-1}$ \\
\hline $\mathrm{Br}^{-}$ & 3.59923 & 1.75728 \\
\hline $\mathrm{I}^{-}$ & 4.18722 & 1.67360 \\
\hline
\end{tabular}



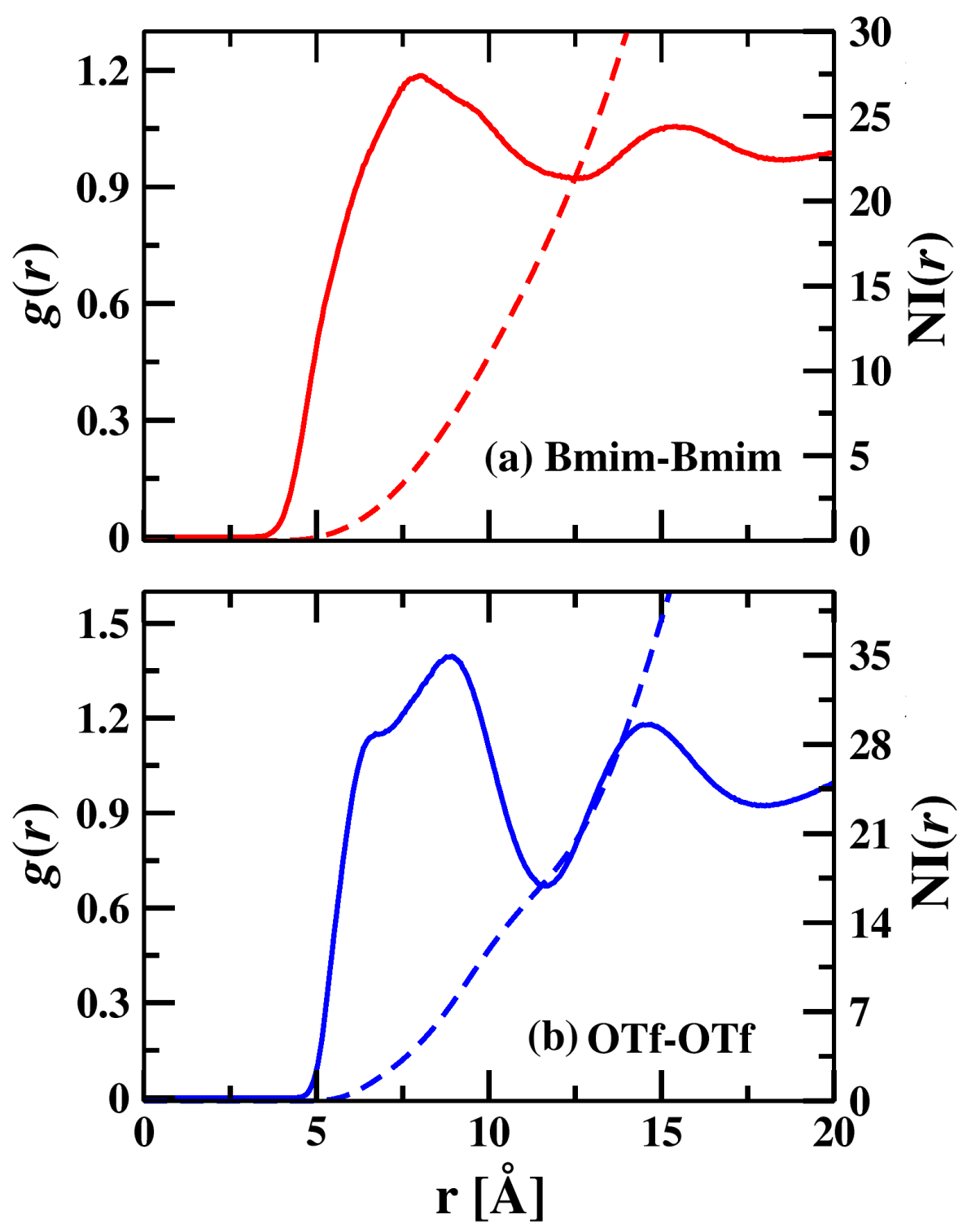

Figure S1. The radial distributions of (a) $[\mathrm{Bmim}]^{+}$around $[\mathrm{Bmim}]^{+}$and (b) OTf around OTf in pure [Bmim][OTf]. 


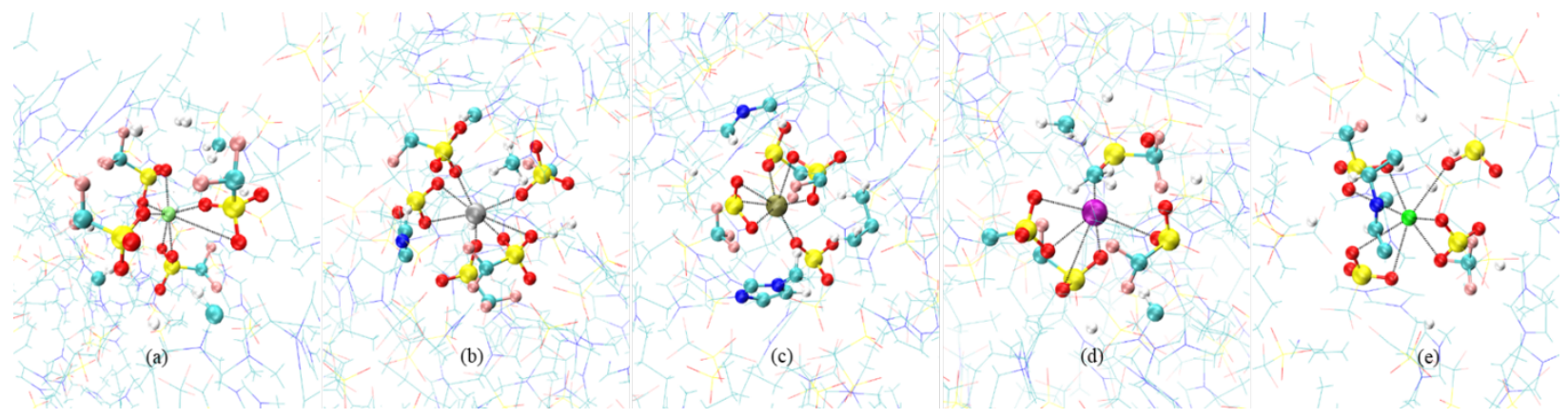

Figure S2. Snapshots of the solvation shell within $5 \AA$ of $\mathrm{Li}$ (a), $\mathrm{Na}$ (b), K (c), Rb (d), and Cs (e) cation in the mixture of single ion pair of alkali metal chlorides and ionic liquid. Lime, silver, tan, purple, and green colour represent the $\mathrm{Li}, \mathrm{Na}, \mathrm{K}, \mathrm{Rb}$, and $\mathrm{Cs}$ cation, respectively. 

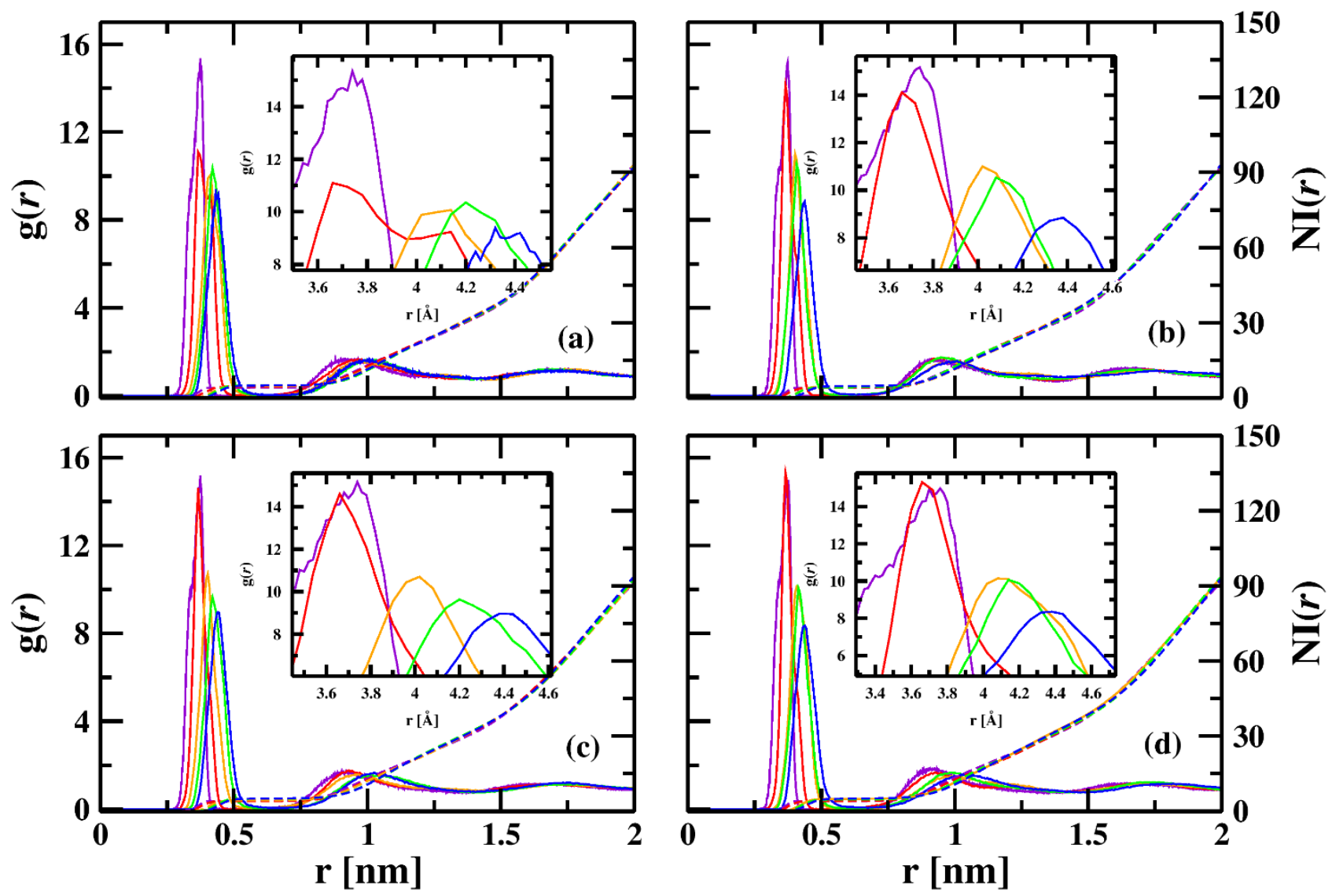

Figure S3. RDFs of OTf anion around $\mathrm{M}^{+}$in the mixture of single ion pair of $\mathrm{MF}$ (a), $\mathrm{MCl}(\mathrm{b}), \mathrm{MBr}(\mathrm{c})$, and MI (d) in IL, where the color violet, red, orange, green, and blue represent the mixture of LiX, NaX, $\mathrm{KX}, \mathrm{RbX}$, and $\mathrm{CsX}(\mathrm{X}=$ halide anion) with ionic liquid. 


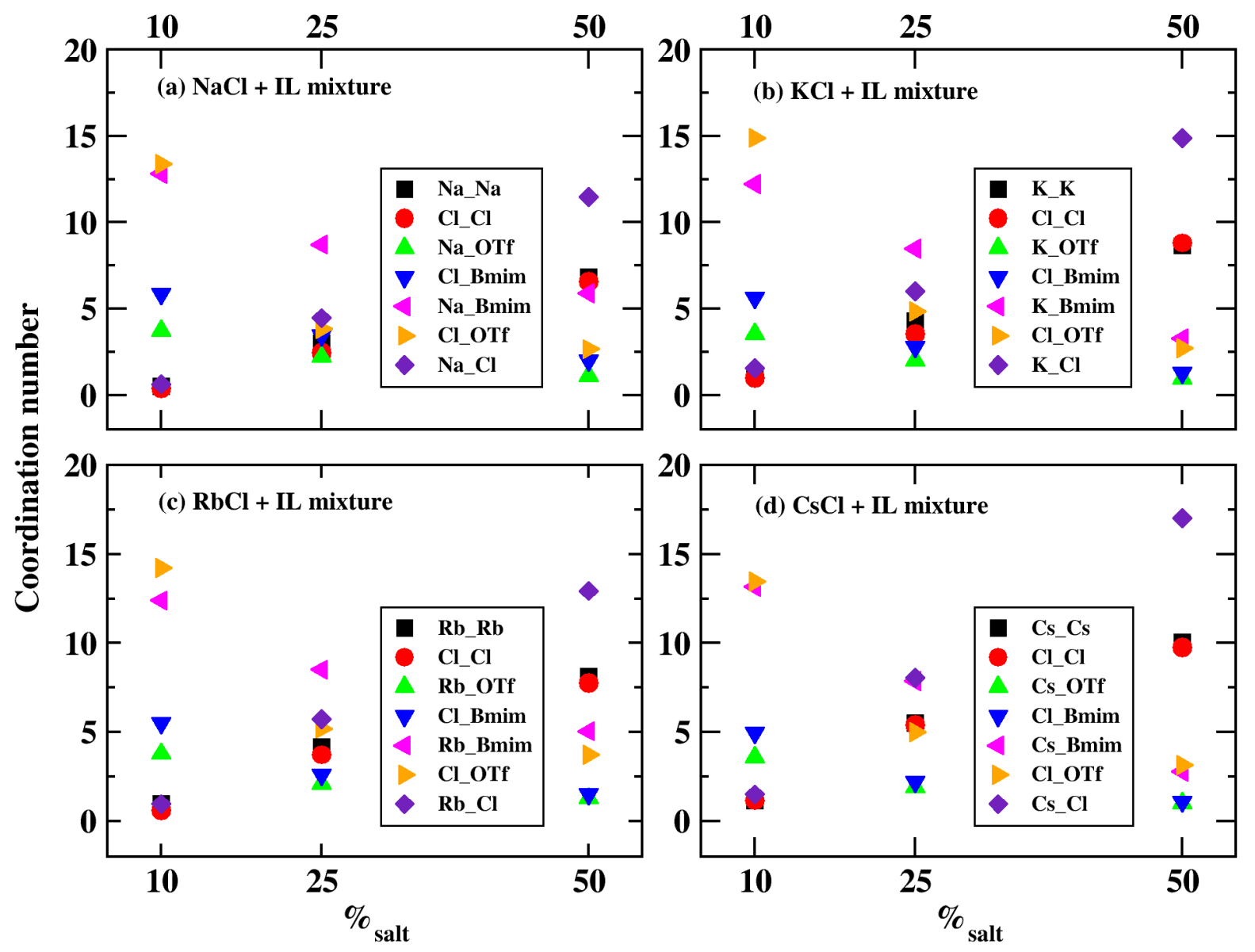

Figure S4. Coordination numbers of different species (a) $\mathrm{NaCl}$ (b) $\mathrm{KCl}$ (c) $\mathrm{RbCl}$ and (d) $\mathrm{CsCl}$ at different concentrations in IL. 

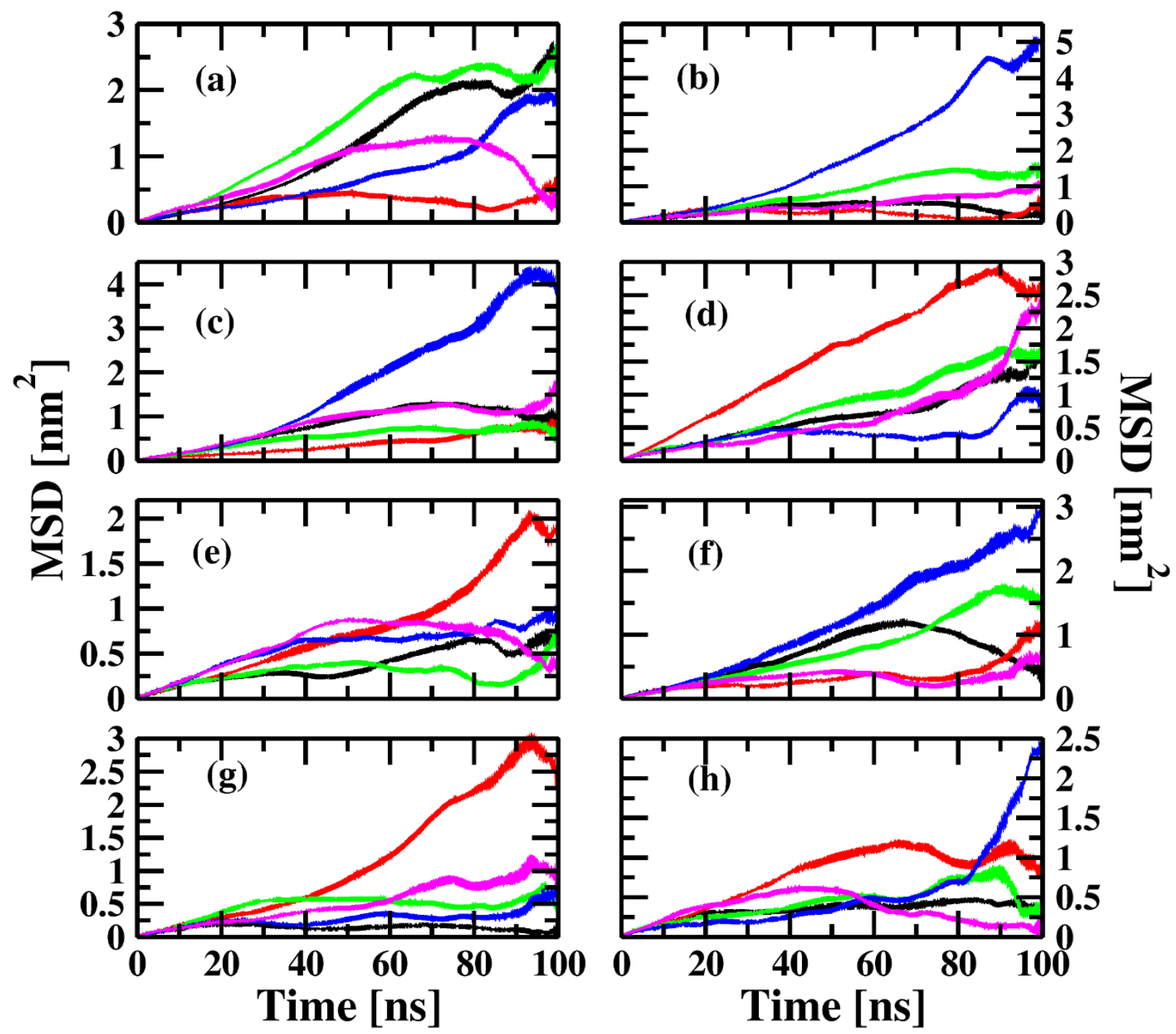

Figure S5. Mean squared displacements of (a) $\mathrm{M}^{+}$and (b) $\mathrm{F}^{-}$in $\mathrm{MF}$, (c) $\mathrm{M}^{+}$and (d) $\mathrm{Cl}^{-}$in $\mathrm{MCl}$, (e) $\mathrm{M}^{+}$and (f) $\mathrm{Br}^{-}$in $\mathrm{MBr}$ and (g) $\mathrm{M}^{+}$and (h) $\mathrm{I}^{-}$in $\mathrm{MI}$ in the mixtures of single ion pair of alkali MX in IL. Black, red, green, blue, and magenta represent $\mathrm{Li}^{+}, \mathrm{Na}^{+}, \mathrm{K}^{+}, \mathrm{Rb}^{+}$, and $\mathrm{Cs}^{+}$, respectively in each of the cases. 

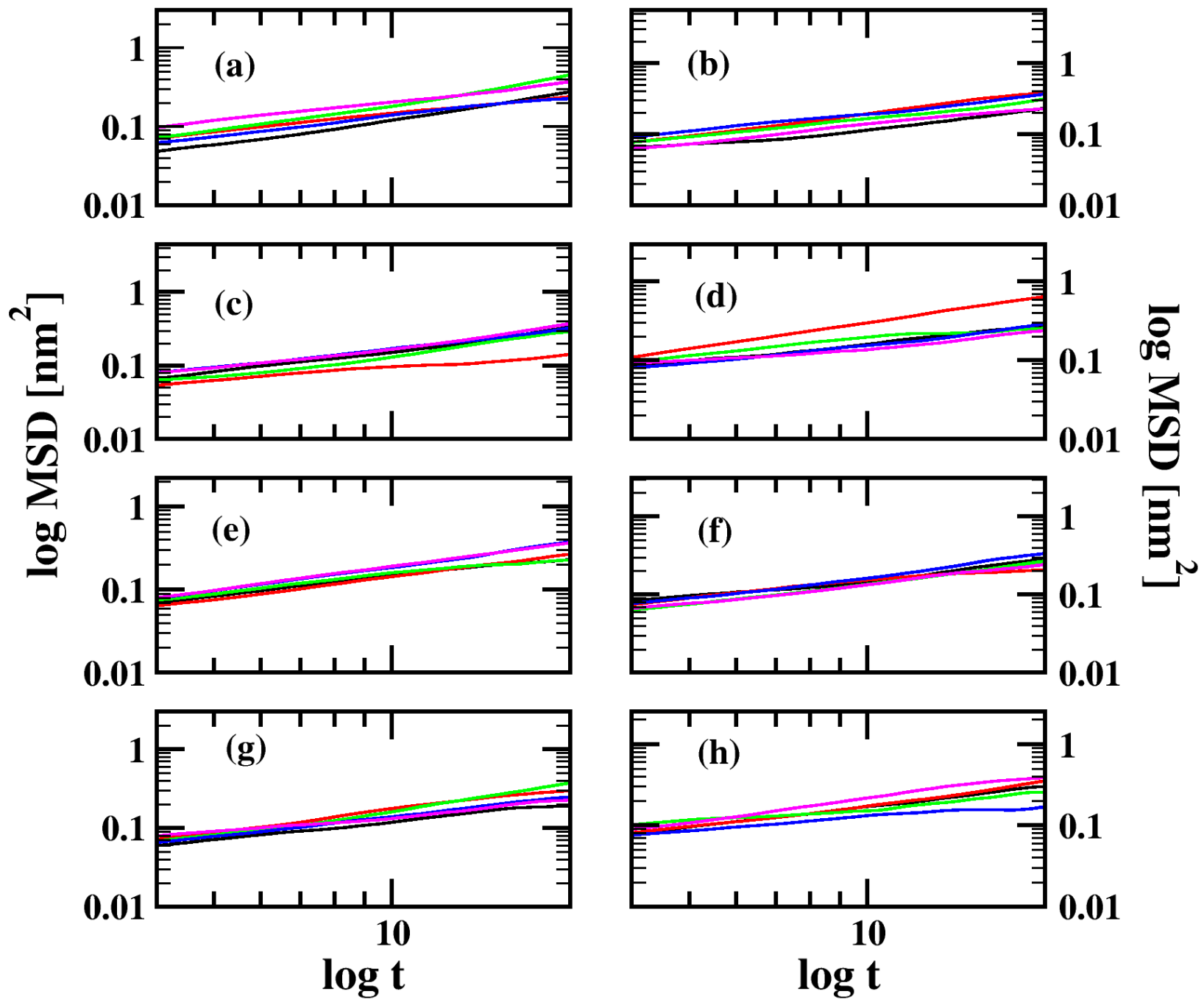

Figure S6. log-log mean squared displacement plots (a) $\mathrm{M}^{+}$and (b) $\mathrm{F}^{-}$in $\mathrm{MF}$, (c) $\mathrm{M}^{+}$and (d) $\mathrm{Cl}^{-}$in $\mathrm{MCl}$, (e) $\mathrm{M}^{+}$and (f) $\mathrm{Br}^{-}$in $\mathrm{MBr}$ and (g) $\mathrm{M}^{+}$and (h) $\mathrm{I}^{-}$in $\mathrm{MI}$ in the mixtures of single ion pair of alkali MX in IL. Black, red, green, blue, and magenta represent $\mathrm{Li}^{+}, \mathrm{Na}^{+}, \mathrm{K}^{+}, \mathrm{Rb}^{+}$, and $\mathrm{Cs}^{+}$, respectively, in each of the cases. 

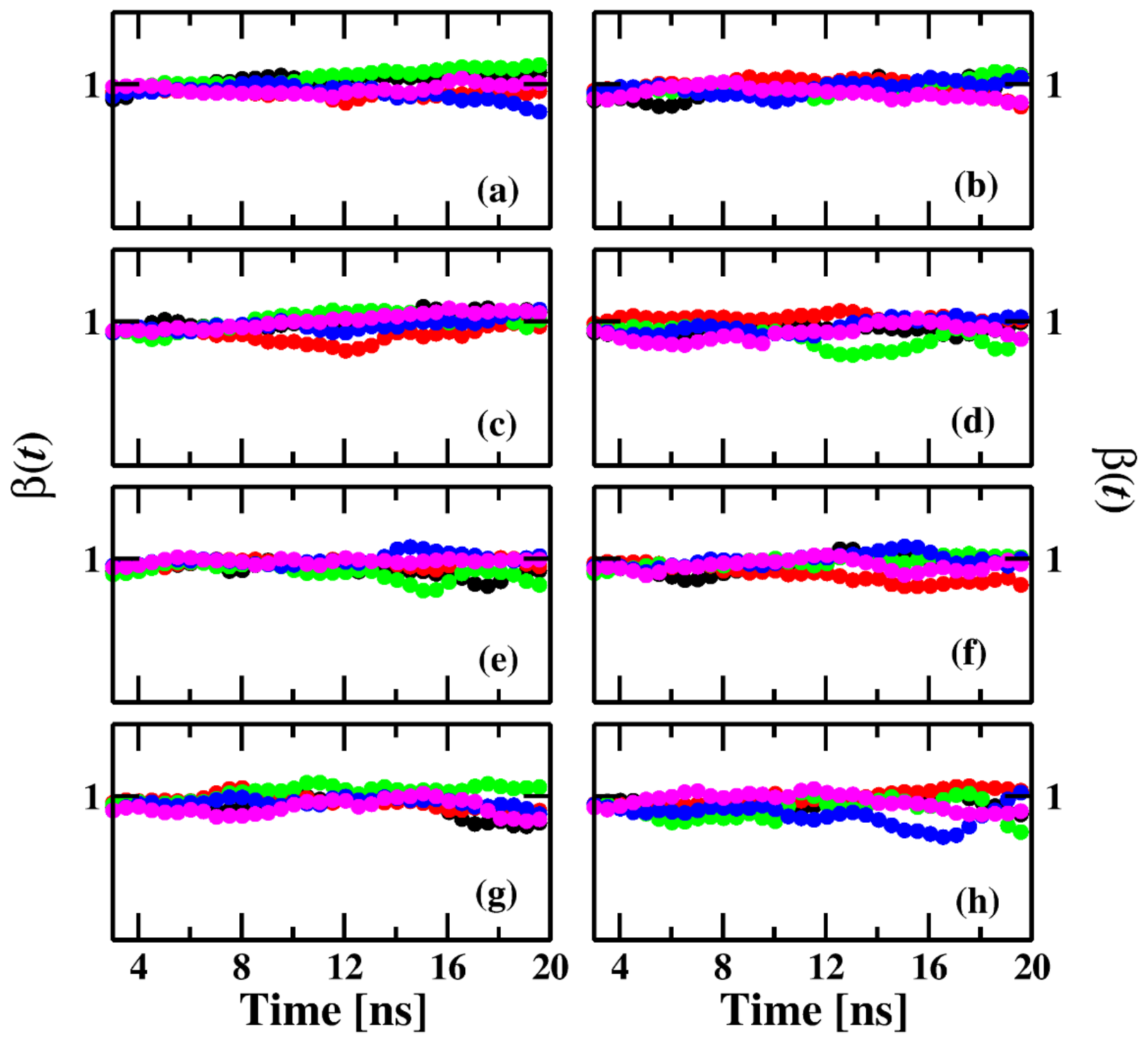

Figure S7. Beta values derived from values of mean squared displacement (a) $\mathrm{M}^{+}$and (b) $\mathrm{F}^{-}$in $\mathrm{MF}$, (c) $\mathrm{M}^{+}$and (d) $\mathrm{Cl}^{-}$in $\mathrm{MCl}$, (e) $\mathrm{M}^{+}$and (f) $\mathrm{Br}^{-}$in $\mathrm{MBr}$ and (g) $\mathrm{M}^{+}$and (h) $\mathrm{I}^{-}$in $\mathrm{MI}$ in the mixtures of single ion pair of alkali MX in IL. Black, red, green, blue, and magenta represent $\mathrm{Li}^{+}, \mathrm{Na}^{+}, \mathrm{K}^{+}, \mathrm{Rb}^{+}$, and $\mathrm{Cs}^{+}$, respectively, in each of the cases. 

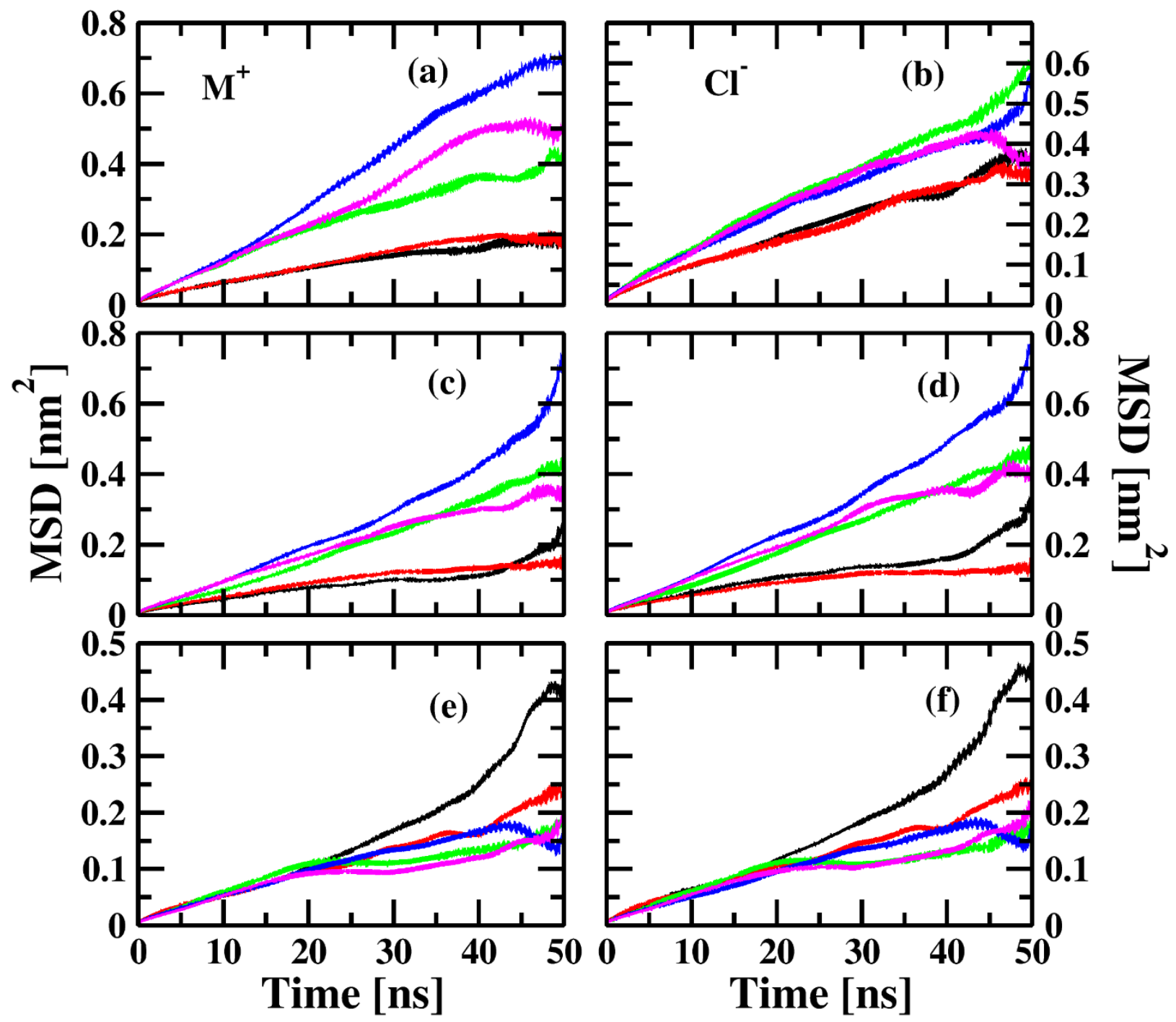

Figure S8. Mean square displacements of single $\mathrm{M}^{+}$and $\mathrm{Cl}^{-}$at (a-b) $10 \%$, (c-d) $25 \%$ and (e-f) $50 \%$ of salt concentration in IL. Black, red, green, blue, and magenta colours represent the mixtures of $\mathrm{LiCl}, \mathrm{NaCl}$, $\mathrm{KCl}, \mathrm{RbCl}$, and $\mathrm{CsCl}$ in IL respectively. 

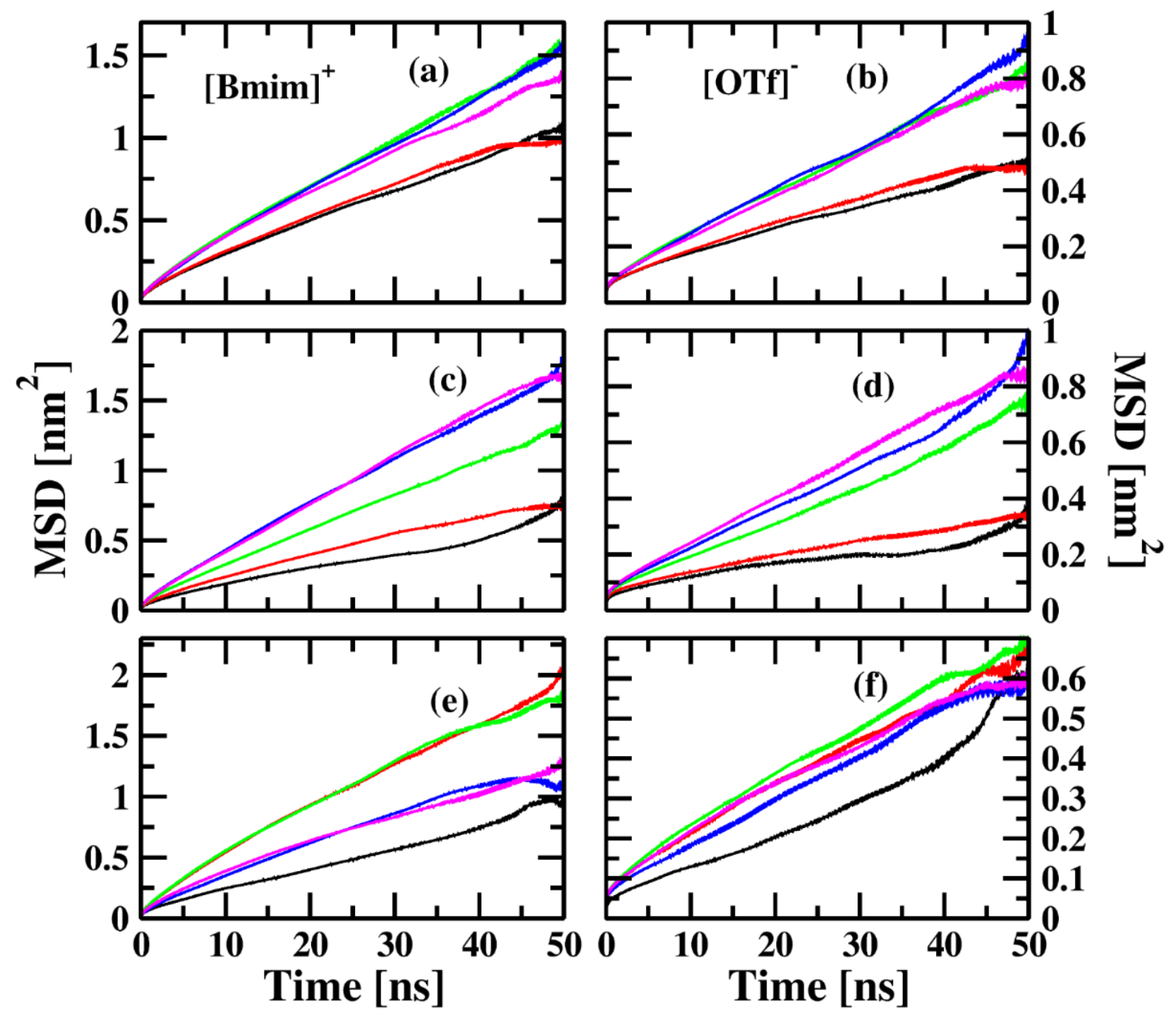

Figure S9. Mean square displacements of (a) $[\mathrm{Bmim}]^{+}$and (b) $[\mathrm{OTf}]^{-}$at $10 \%$, (c) $[\mathrm{Bmim}]^{+}$and (d) $[\mathrm{OTf}]^{-}$ at $25 \%$, and (e) $[\mathrm{Bmim}]^{+}$and (f) $[\mathrm{OTf}]^{-}$at $50 \%$ salt concentration. Black, red, green, blue, and magenta colours represent the mixtures of $\mathrm{LiCl}, \mathrm{NaCl}, \mathrm{KCl}, \mathrm{RbCl}$, and $\mathrm{CsCl}$ in IL respectively. 

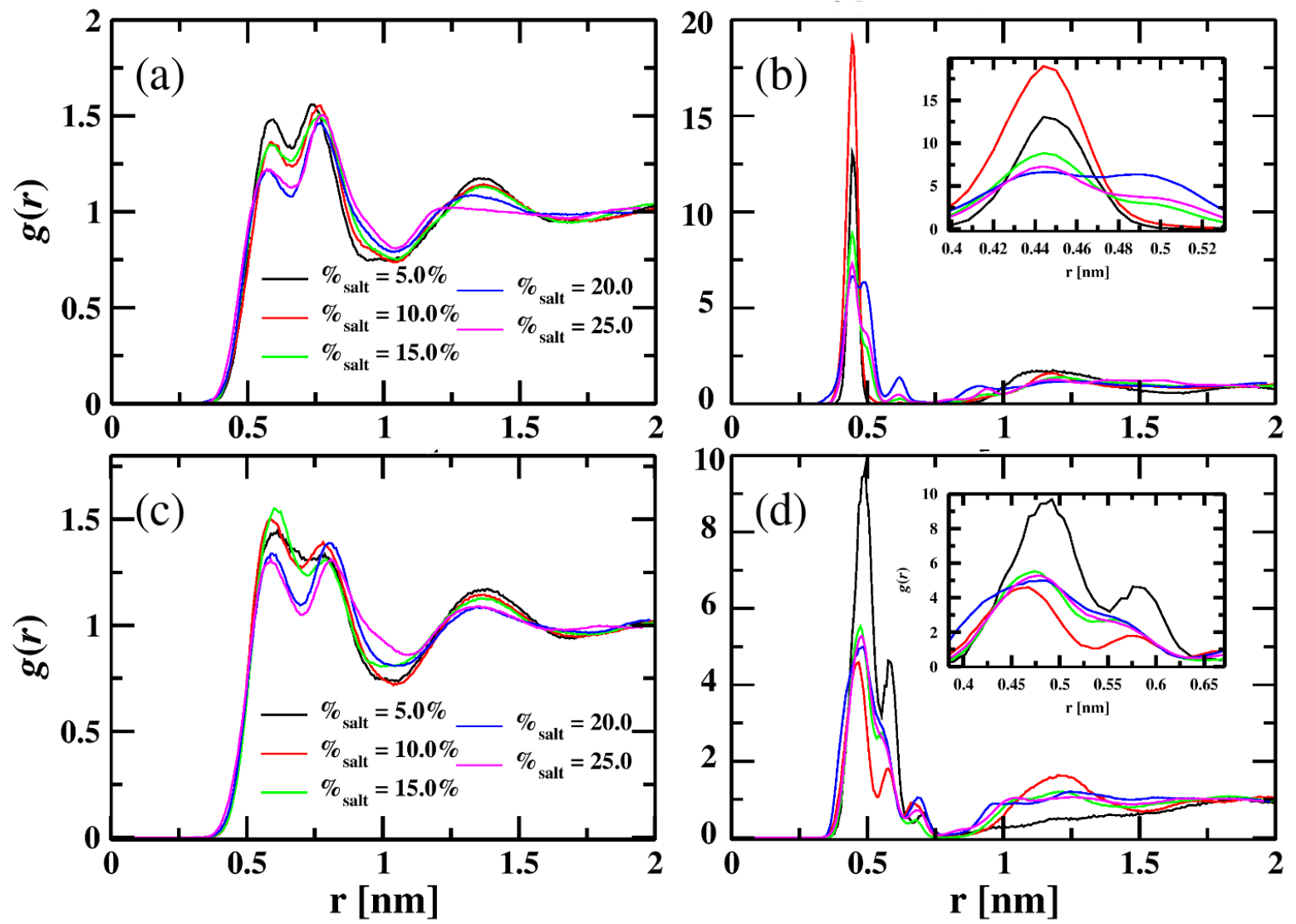

Figure S10. RDF of (a) $\mathrm{Li}^{+}-[\mathrm{Bmim}]^{+}$(b) $\mathrm{Li}^{+}-\mathrm{Li}^{+}$, (c) $\mathrm{Na}^{+}-[\mathrm{Bmim}]^{+}$and (d) $\mathrm{Na}^{+}-\mathrm{Na}^{+}$pairs 

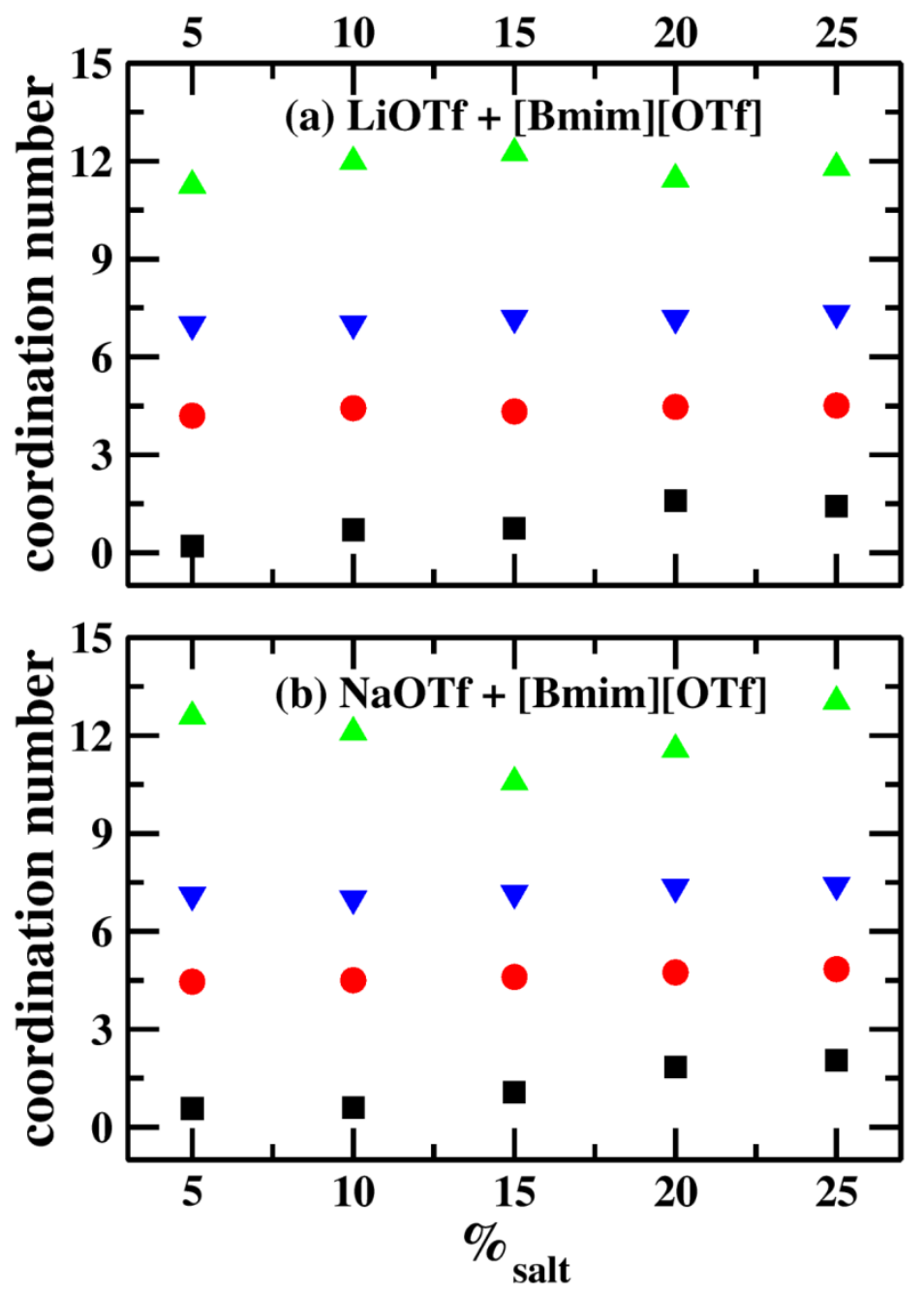

Figure S11. Coordination numbers of metal cations (black squares), OTf anions (red circles), and Bmim cation (green triangles) around a metal cation in solutions of IL with Li[OTf] (a) and Na[OTf] (b), respectively. The numbers of OTf anions around Bmim cation (blue triangles) are also included. 

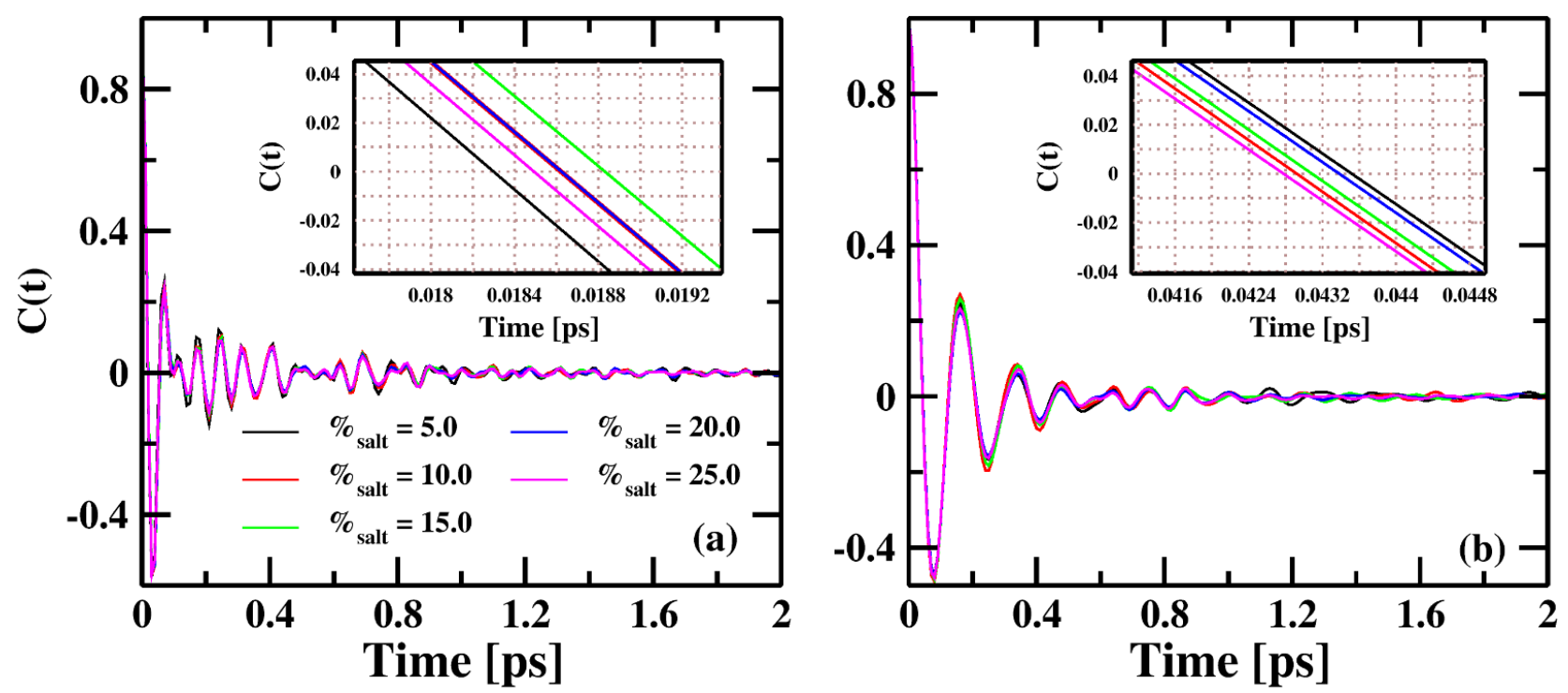

Figure S12. Concentration dependence of lithium (a) and sodium (b) VACFs in Li[OTf] and Na[OTf] mixed with IL, respectively. The insets shows the evolution of collision time. 

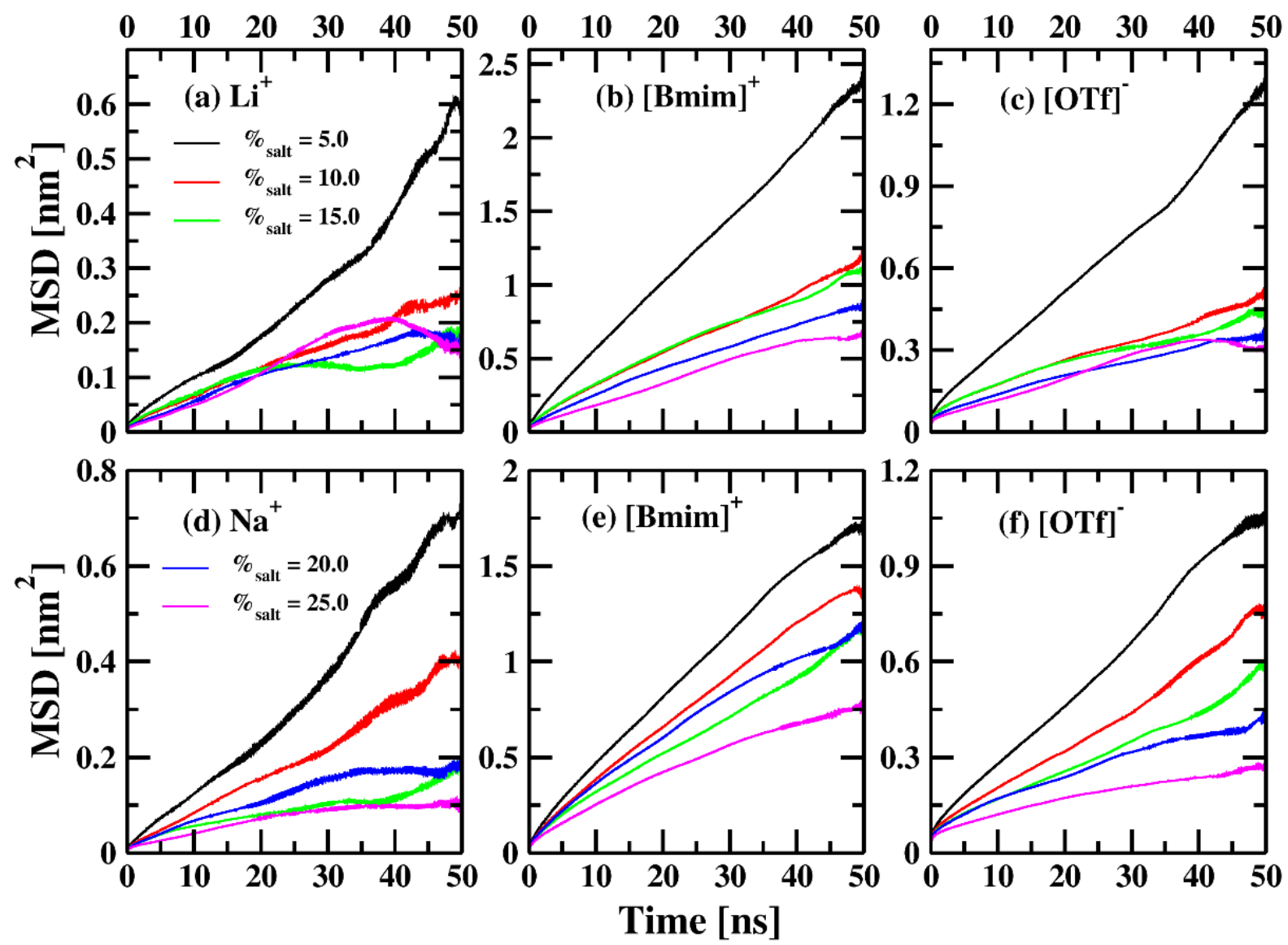

Figure S13. MSDs of (a) $\mathrm{Li}^{+}$, (b) $[\mathrm{Bmim}]^{+}$and (c) $[\mathrm{OTf}]^{-}$in the mixture of $\mathrm{Li}[\mathrm{OTf}]$ in IL and (d) $\mathrm{Na}^{+}$, (e) $[\mathrm{Bmim}]^{+}$and (f) $[\mathrm{OTf}]^{-}$in the mixture of $\mathrm{Na}[\mathrm{OTf}]$ in IL, respectively. The different colours represent different concentrations of the salt in IL, as shown in the legends. 

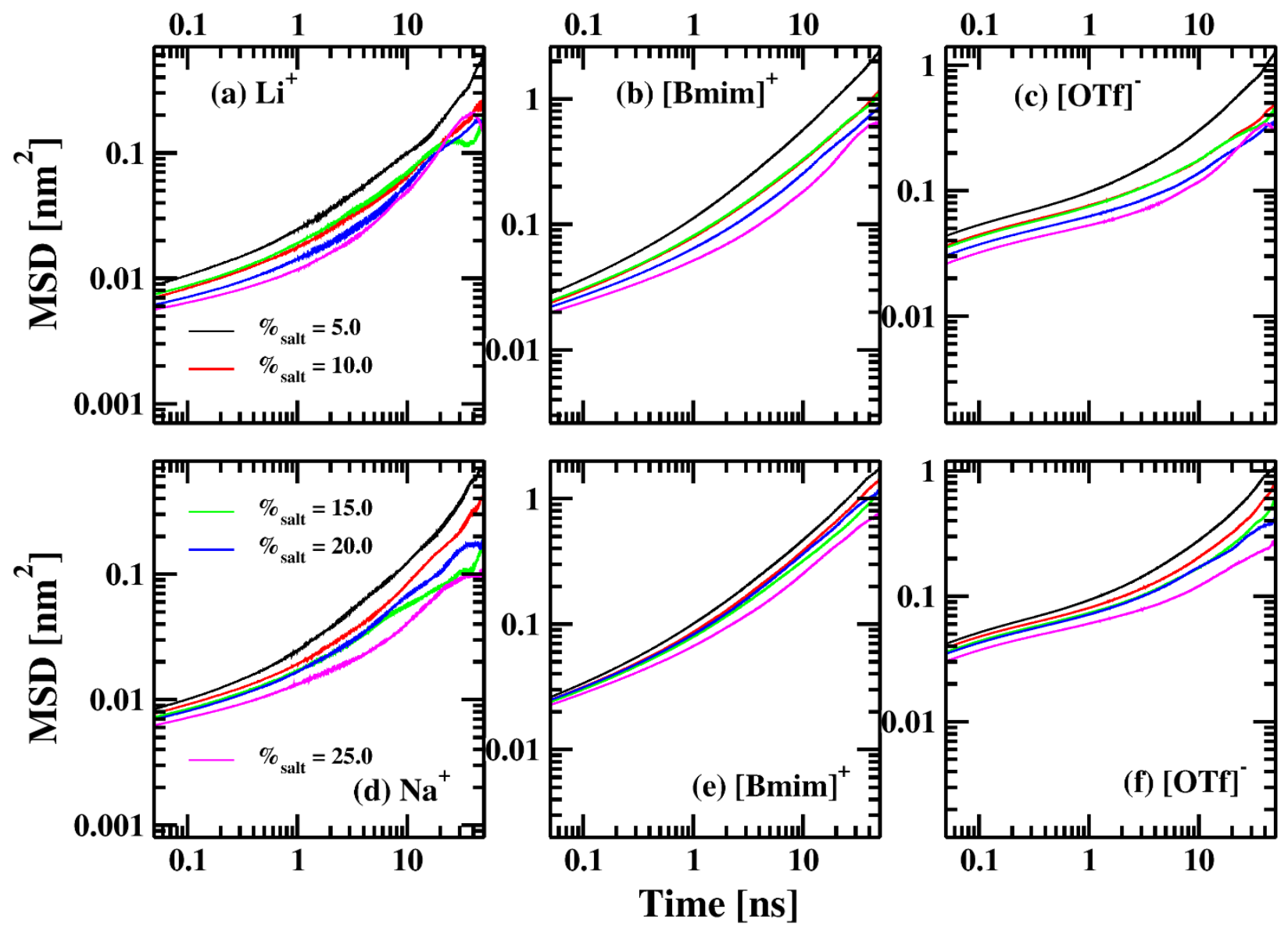

Figure S14. The log-log plot of MSDs of (a) $\mathrm{Li}^{+}$, (b) $[\mathrm{Bmim}]^{+}$and (c) $[\mathrm{OTf}]^{-}$in the mixture of Li[OTf] in IL and (d) $\mathrm{Na}^{+}$, (e) $[\mathrm{Bmim}]^{+}$and (f) $[\mathrm{OTf}]^{-}$in the mixture of $\mathrm{Na}[\mathrm{OTf}]$ in IL, respectively. The different colours represent different concentrations of the salt in IL, as shown in the legends. 

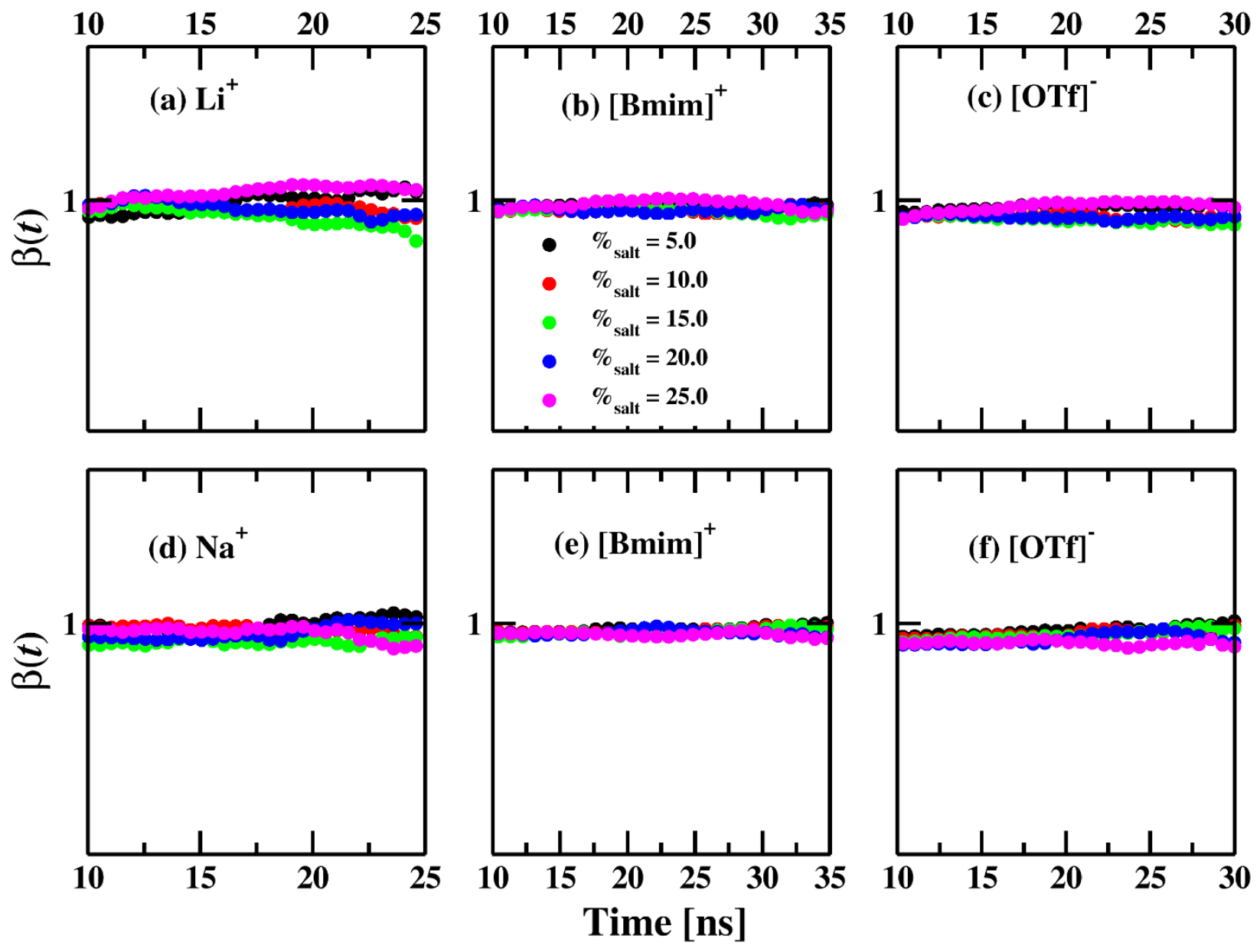

Figure S15. The beta values derived from MSDs of (a) $\mathrm{Li}^{+}$, (b) $[\mathrm{Bmim}]^{+}$and (c) $[\mathrm{OTf}]^{-}$in the mixture of $\mathrm{Li}[\mathrm{OTf}]$ in IL and (d) $\mathrm{Na}^{+}$, (e) [Bmim] $]^{+}$and (f) [OTf] in the mixture of $\mathrm{Na}[\mathrm{OTf}]$ in IL, respectively. The different colours represent different concentrations of the salt in IL, as shown in the legends. 


\section{GROMACS topology file for $[\mathrm{Bmim}]^{+}$:}

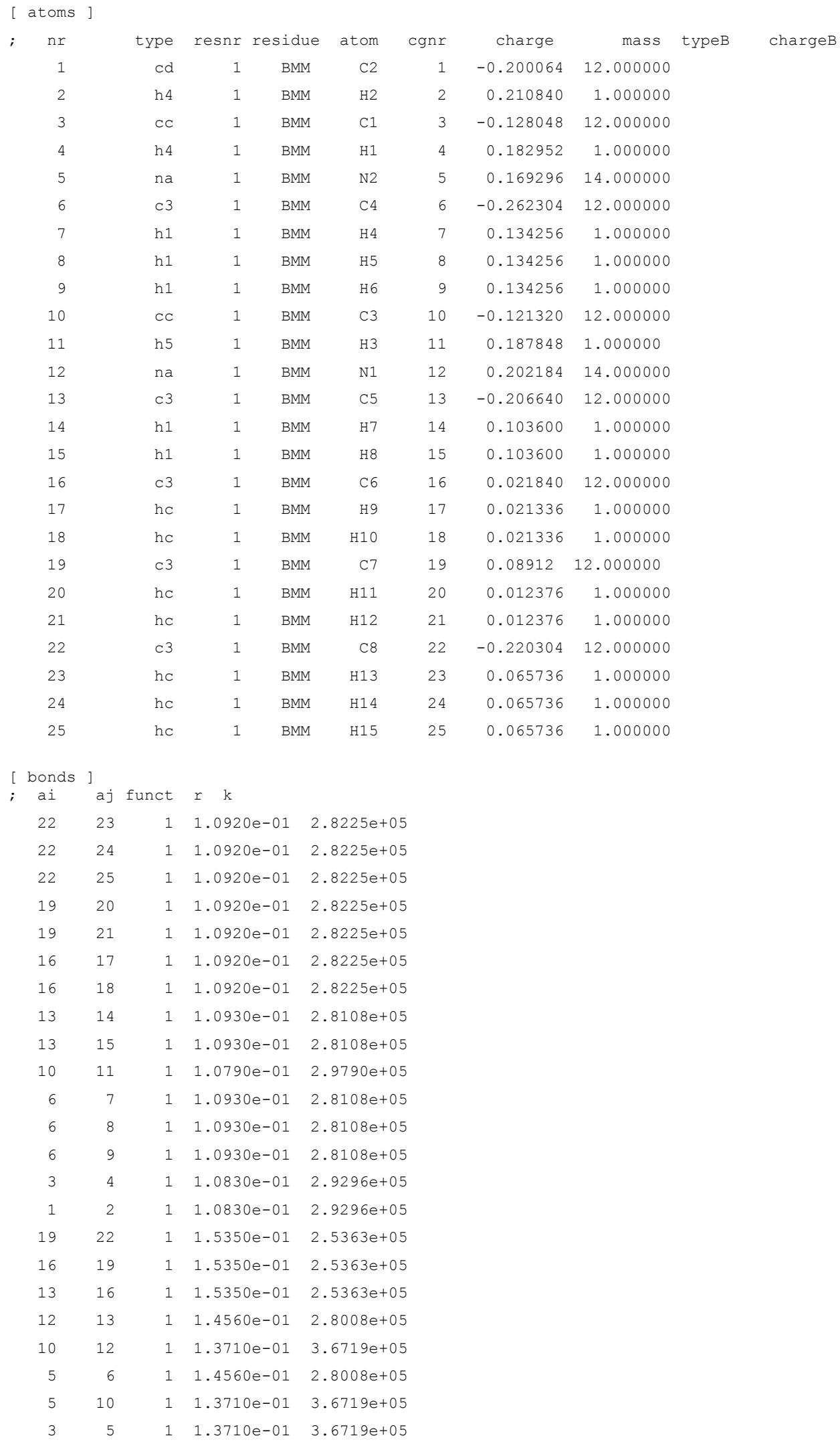




\begin{tabular}{|c|c|c|c|}
\hline \multirow{2}{*}{$\begin{array}{l}1 \\
1\end{array}$} & 3 & \multirow{2}{*}{$\begin{array}{l}1.3710 \mathrm{e}-01 \\
1.3710 \mathrm{e}-01\end{array}$} & \multirow{2}{*}{$\begin{array}{l}4.2175 e+05 \\
3.6719 e+05\end{array}$} \\
\hline & 12 & & \\
\hline \multicolumn{4}{|c|}{ [ pairs ] } \\
\hline ai & aj funct & & \\
\hline 21 & 23 & 1 & \\
\hline 21 & 24 & 1 & \\
\hline 21 & 25 & 1 & \\
\hline 20 & 23 & 1 & \\
\hline 20 & 24 & 1 & \\
\hline 20 & 25 & 1 & \\
\hline 18 & 20 & 1 & \\
\hline 18 & 21 & 1 & \\
\hline 18 & 22 & 1 & \\
\hline 17 & 20 & 1 & \\
\hline 17 & 21 & 1 & \\
\hline 17 & 22 & 1 & \\
\hline 16 & 23 & 1 & \\
\hline 16 & 24 & 1 & \\
\hline 16 & 25 & 1 & \\
\hline 15 & 17 & 1 & \\
\hline 15 & 18 & 1 & \\
\hline 15 & 19 & 1 & \\
\hline 14 & 17 & 1 & \\
\hline 14 & 18 & 1 & \\
\hline 14 & 19 & 1 & \\
\hline 13 & 20 & 1 & \\
\hline 13 & 21 & 1 & \\
\hline 12 & 17 & 1 & \\
\hline 12 & 18 & 1 & \\
\hline 11 & 13 & 1 & \\
\hline 10 & 14 & 1 & \\
\hline 10 & 15 & 1 & \\
\hline 9 & 10 & 1 & \\
\hline 8 & 10 & 1 & \\
\hline 7 & 10 & 1 & \\
\hline 6 & 11 & 1 & \\
\hline 12 & 4 & 1 & \\
\hline 4 & 6 & 1 & \\
\hline 4 & 10 & 1 & \\
\hline 3 & 7 & 1 & \\
\hline 3 & 8 & 1 & \\
\hline 3 & 9 & 1 & \\
\hline 3 & 11 & 1 & \\
\hline 2 & 4 & 1 & \\
\hline 2 & 5 & 1 & \\
\hline 2 & 10 & 1 & \\
\hline 2 & 13 & 1 & \\
\hline 1 & 11 & 1 & \\
\hline 1 & 14 & 1 & \\
\hline 1 & 15 & 1 & \\
\hline 13 & 22 & 1 & \\
\hline 12 & 19 & 1 & \\
\hline 10 & 16 & 1 & \\
\hline
\end{tabular}




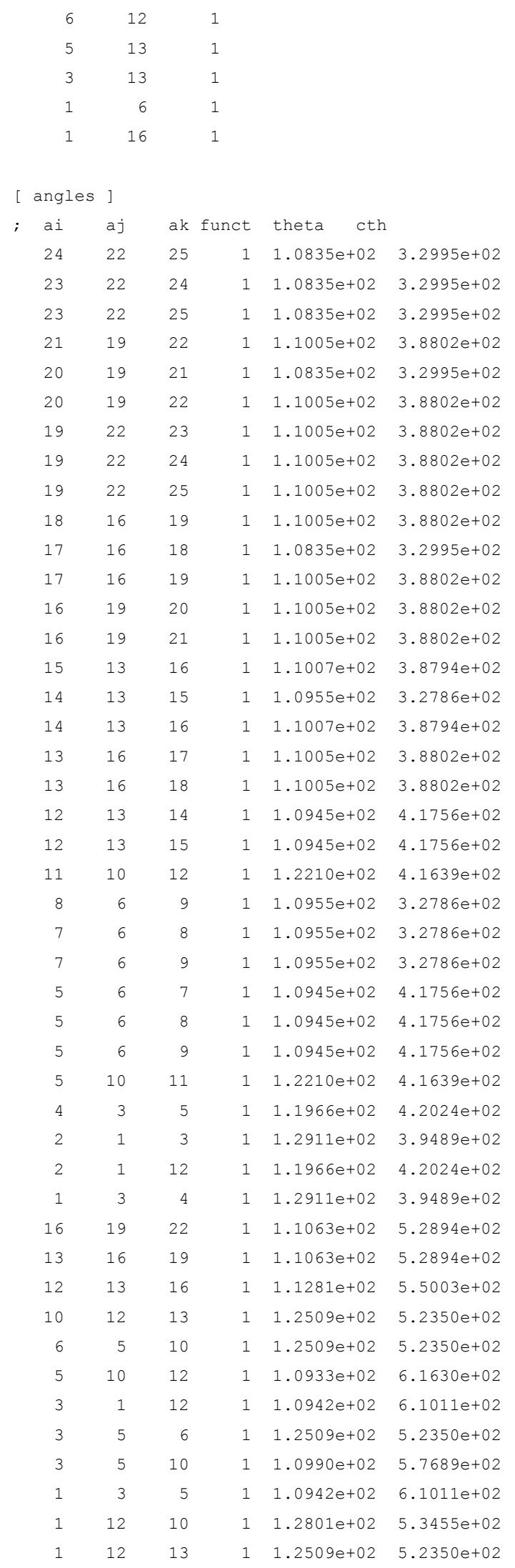




\begin{tabular}{|c|c|c|c|c|c|c|c|c|c|c|}
\hline$j$ & k 1 & & func & $\mathrm{CO}$ & C5 & & & & & \\
\hline 21 & 19 & 22 & 23 & 3 & 0.62760 & 1.88280 & 0.00000 & -2.51040 & 0.00000 & 0.00000 \\
\hline 21 & 19 & 22 & 24 & 3 & 0.62760 & 1.88280 & 0.00000 & -2.51040 & 0.00000 & 0.00000 \\
\hline 21 & 19 & 22 & 25 & 3 & 0.62760 & 1.88280 & 0.00000 & -2.51040 & 0.00000 & 0.00000 \\
\hline 20 & 19 & 22 & 23 & 3 & 0.62760 & 1.88280 & 0.00000 & -2.51040 & 0.00000 & 0.00000 \\
\hline 20 & 19 & 22 & 24 & 3 & 0.62760 & 1.88280 & 0.00000 & -2.51040 & 0.00000 & 0.00000 \\
\hline 20 & 19 & 22 & 25 & 3 & 0.62760 & 1.88280 & 0.00000 & -2.51040 & 0.00000 & 0.00000 \\
\hline 18 & 16 & 19 & 20 & 3 & 0.62760 & 1.88280 & 0.00000 & -2.51040 & 0.00000 & 0.00000 \\
\hline 18 & 16 & 19 & 21 & 3 & 0.62760 & 1.88280 & 0.00000 & -2.51040 & 0.00000 & 0.00000 \\
\hline 18 & 16 & 19 & 22 & 3 & 0.66944 & 2.00832 & 0.00000 & -2.67776 & 0.00000 & 0.00000 \\
\hline 17 & 16 & 19 & 20 & 3 & 0.62760 & 1.88280 & 0.00000 & -2.51040 & 0.00000 & 0.00000 \\
\hline 17 & 16 & 19 & 21 & 3 & 0.62760 & 1.88280 & 0.00000 & -2.51040 & 0.00000 & 0.00000 \\
\hline 17 & 16 & 19 & 22 & 3 & 0.66944 & 2.00832 & 0.00000 & -2.67776 & 0.00000 & 0.00000 \\
\hline 16 & 19 & 22 & 23 & 3 & 0.66944 & 2.00832 & 0.00000 & -2.67776 & 0.00000 & 0.00000 \\
\hline 16 & 19 & 22 & 24 & 3 & 0.66944 & 2.00832 & 0.00000 & -2.67776 & 0.00000 & 0.00000 \\
\hline 16 & 19 & 22 & 25 & 3 & 0.66944 & 2.00832 & 0.00000 & -2.67776 & 0.00000 & 0.00000 \\
\hline 15 & 13 & 16 & 17 & 3 & 0.65270 & 1.95811 & 0.00000 & -2.61082 & 0.00000 & 0.00000 \\
\hline 15 & 13 & 16 & 18 & 3 & 0.65270 & 1.95811 & 0.00000 & -2.61082 & 0.00000 & 0.00000 \\
\hline 15 & 13 & 16 & 19 & 3 & 0.65270 & 1.95811 & 0.00000 & -2.61082 & 0.00000 & 0.00000 \\
\hline 14 & 13 & 16 & 17 & 3 & 0.65270 & 1.95811 & 0.00000 & -2.61082 & 0.00000 & 0.00000 \\
\hline 14 & 13 & 16 & 18 & 3 & 0.65270 & 1.95811 & 0.00000 & -2.61082 & 0.00000 & 0.00000 \\
\hline 14 & 13 & 16 & 19 & 3 & 0.65270 & 1.95811 & 0.00000 & -2.61082 & 0.00000 & 0.00000 \\
\hline 13 & 16 & 19 & 20 & 3 & 0.66944 & 2.00832 & 0.00000 & -2.67776 & 0.00000 & 0.00000 \\
\hline 13 & 16 & 19 & 21 & 3 & 0.66944 & 2.00832 & 0.00000 & -2.67776 & 0.00000 & 0.00000 \\
\hline 12 & 13 & 16 & 17 & 3 & 0.65270 & 1.95811 & 0.00000 & -2.61082 & 0.00000 & 0.00000 \\
\hline 12 & 13 & 16 & 18 & 3 & 0.65270 & 1.95811 & 0.00000 & -2.61082 & 0.00000 & 0.00000 \\
\hline 11 & 10 & 12 & 13 & 3 & 14.22560 & 0.00000 & -14.22560 & 0.00000 & 0.00000 & 0.00000 \\
\hline 10 & 12 & 13 & 14 & 3 & 0.00000 & 0.00000 & 0.00000 & 0.00000 & 0.00000 & 0.00000 \\
\hline 10 & 12 & 13 & 15 & 3 & 0.00000 & 0.00000 & 0.00000 & 0.00000 & 0.00000 & 0.00000 \\
\hline 9 & 6 & 5 & 10 & 3 & 0.00000 & 0.00000 & 0.00000 & 0.00000 & 0.00000 & 0.00000 \\
\hline 8 & 6 & 5 & 10 & 3 & 0.00000 & 0.00000 & 0.00000 & 0.00000 & 0.00000 & 0.00000 \\
\hline 7 & 6 & 5 & 10 & 3 & 0.00000 & 0.00000 & 0.00000 & 0.00000 & 0.00000 & 0.00000 \\
\hline 6 & 5 & 10 & 11 & 3 & 14.22560 & 0.00000 & -14.22560 & 0.00000 & 0.00000 & 0.00000 \\
\hline 12 & 1 & 3 & 4 & 3 & 33.47200 & 0.00000 & -33.47200 & 0.00000 & 0.00000 & 0.00000 \\
\hline 4 & 3 & 5 & 6 & 3 & 14.22560 & 0.00000 & -14.22560 & 0.00000 & 0.00000 & 0.00000 \\
\hline 4 & 3 & 5 & 10 & 3 & 14.22560 & 0.00000 & -14.22560 & 0.00000 & 0.00000 & 0.00000 \\
\hline 3 & 5 & 6 & 7 & 3 & 0.00000 & 0.00000 & 0.00000 & 0.00000 & 0.00000 & 0.00000 \\
\hline 3 & 5 & 6 & 8 & 3 & 0.00000 & 0.00000 & 0.00000 & 0.00000 & 0.00000 & 0.00000 \\
\hline 3 & 5 & 6 & 9 & 3 & 0.00000 & 0.00000 & 0.00000 & 0.00000 & 0.00000 & 0.00000 \\
\hline 3 & 5 & 10 & 11 & 3 & 14.22560 & 0.00000 & -14.22560 & 0.00000 & 0.00000 & 0.00000 \\
\hline 2 & 1 & 3 & 4 & 3 & 33.47200 & 0.00000 & -33.47200 & 0.00000 & 0.00000 & 0.00000 \\
\hline 2 & 1 & 3 & 5 & 3 & 33.47200 & 0.00000 & -33.47200 & 0.00000 & 0.00000 & 0.00000 \\
\hline 2 & 1 & 12 & 10 & 3 & 14.22560 & 0.00000 & -14.22560 & 0.00000 & 0.00000 & 0.00000 \\
\hline 2 & 1 & 12 & 13 & 3 & 14.22560 & 0.00000 & -14.22560 & 0.00000 & 0.00000 & 0.00000 \\
\hline 1 & 12 & 10 & 11 & 3 & 14.22560 & 0.00000 & -14.22560 & 0.00000 & 0.00000 & 0.00000 \\
\hline 1 & 12 & 13 & 14 & 3 & 0.00000 & 0.00000 & 0.00000 & 0.00000 & 0.00000 & 0.00000 \\
\hline 1 & 12 & 13 & 15 & 3 & 0.00000 & 0.00000 & 0.00000 & 0.00000 & 0.00000 & 0.00000 \\
\hline 11 & 5 & 10 & 12 & 3 & 9.20480 & 0.00000 & -9.20480 & 0.00000 & 0.00000 & 0.00000 \\
\hline 1 & 4 & 3 & 5 & 3 & 9.20480 & 0.00000 & -9.20480 & 0.00000 & 0.00000 & 0.00000 \\
\hline 12 & 1 & 2 & 3 & 3 & 9.20480 & 0.00000 & -9.20480 & 0.00000 & 0.00000 & 0.00000 \\
\hline 13 & 16 & 19 & 22 & 3 & 3.68192 & 3.09616 & -2.09200 & -3.01248 & 0.00000 & 0.00000 \\
\hline 12 & 13 & 16 & 19 & 3 & 0.65270 & 1.95811 & 0.00000 & -2.61082 & 0.00000 & 0.00000 \\
\hline 10 & 12 & 13 & 16 & 3 & 0.00000 & 0.00000 & 0.00000 & 0.00000 & 0.00000 & 0.00000 \\
\hline
\end{tabular}




$\begin{array}{llllllllllll}6 & 5 & 10 & 12 & 3 & 14.22560 & 0.00000 & -14.22560 & 0.00000 & 0.00000 & 0.00000 & ; \\ 12 & 1 & 3 & 5 & 3 & 33.47200 & 0.00000 & -33.47200 & 0.00000 & 0.00000 & 0.00000 & ; \\ 5 & 10 & 12 & 13 & 3 & 14.22560 & 0.00000 & -14.22560 & 0.00000 & 0.00000 & 0.00000 & ; \\ 3 & 1 & 12 & 10 & 3 & 14.22560 & 0.00000 & -14.22560 & 0.00000 & 0.00000 & 0.00000 & ; \\ 3 & 1 & 12 & 13 & 3 & 14.22560 & 0.00000 & -14.22560 & 0.00000 & 0.00000 & 0.00000 & ; \\ 3 & 5 & 10 & 12 & 3 & 14.22560 & 0.00000 & -14.22560 & 0.00000 & 0.00000 & 0.00000 & ; \\ 1 & 3 & 5 & 6 & 3 & 14.22560 & 0.00000 & -14.22560 & 0.00000 & 0.00000 & 0.00000 & ; \\ 1 & 3 & 5 & 10 & 3 & 14.22560 & 0.00000 & -14.22560 & 0.00000 & 0.00000 & 0.00000 & ; \\ 1 & 12 & 10 & 5 & 3 & 14.22560 & 0.00000 & -14.22560 & 0.00000 & 0.00000 & 0.00000 & ; \\ 1 & 12 & 13 & 16 & 3 & 0.00000 & 0.00000 & 0.00000 & 0.00000 & 0.00000 & 0.00000 & ; \\ 1 & 12 & 10 & 13 & 3 & 9.20480 & 0.00000 & -9.20480 & 0.00000 & 0.00000 & 0.00000 & ; \\ 6 & 3 & 5 & 10 & 3 & 9.20480 & 0.00000 & -9.20480 & 0.00000 & 0.00000 & 0.00000 & ;\end{array}$


GROMACS topology file for [OTf]':

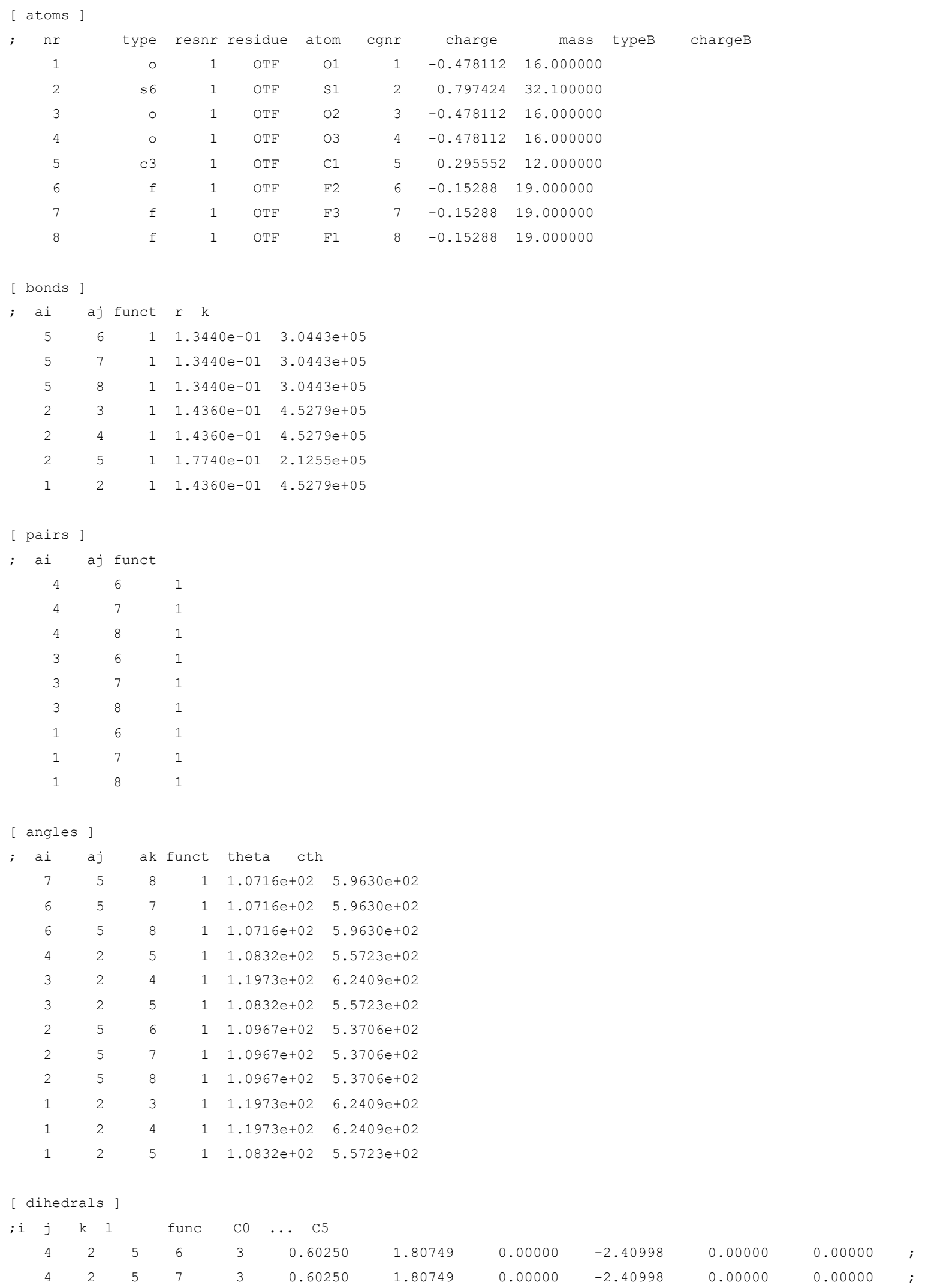




$\begin{array}{lllllllllllll}4 & 2 & 5 & 8 & 3 & 0.60250 & 1.80749 & 0.00000 & -2.40998 & 0.00000 & 0.00000 & ; \\ 3 & 2 & 5 & 6 & 3 & 0.60250 & 1.80749 & 0.00000 & -2.40998 & 0.00000 & 0.00000 & ; \\ 3 & 2 & 5 & 7 & 3 & 0.60250 & 1.80749 & 0.00000 & -2.40998 & 0.00000 & 0.00000 & ; \\ 3 & 2 & 5 & 8 & 3 & 0.60250 & 1.80749 & 0.00000 & -2.40998 & 0.00000 & 0.00000 & ; \\ 1 & 2 & 5 & 6 & 3 & 0.60250 & 1.80749 & 0.00000 & -2.40998 & 0.00000 & 0.00000 & ; \\ 1 & 2 & 5 & 7 & 3 & 0.60250 & 1.80749 & 0.00000 & -2.40998 & 0.00000 & 0.00000 & ; \\ 1 & 2 & 5 & 8 & 3 & 0.60250 & 1.80749 & 0.00000 & -2.40998 & 0.00000 & 0.00000 & ;\end{array}$

